\title{
Investigation of anal function in patients with systemic sclerosis
}

\author{
A L Herrick, J D Barlow, A Bowden, N Williams, A R Hobson, M Irving, M I V Jayson
}

\begin{abstract}
Objective-To investigate anorectal function in women patients with systemic sclerosis (SSc), with and without lower gastrointestinal symptoms.

Methods-Anorectal manometry was performed in 16 patients with SSc: six with no or minimal bowel symptoms, seven with constipation, and three with diarrhoea and faecal incontinence. Eleven healthy women acted as control subjects. Pressure data were recorded via an eight lumen polyvinylchloride water perfused catheter. Station and rapid pull through techniques were used.

Results-In the patients with SSc, mean resting pressure, maximal voluntary squeeze effort, and squeeze vector volume were lower, and squeeze asymmetry was greater, compared with the healthy controls. Differences were significant in the subgroup with constipation.

Conclusion-Radial asymmetry and vector volume parameters provide detailed analysis of segmental anal canal function. Our findings suggest significant segmental deficits in those patients with SSc who have lower gastrointestinal symptoms. The trend towards smaller pressures and squeeze vector volumes in the asymptomatic SSc group suggests subclinical dysfunction in these patients.
\end{abstract}

(Ann Rheum Dis 1996; 55: 370-374)

Upper gastrointestinal dysmotility is a well recognised feature of systemic sclerosis (SSc). Colonic and anorectal function have been less closely studied, though lower bowel dysmotility is known to occur and a proportion of patients are symptomatic for this feature. ${ }^{12}$ The aim of this study was to make a detailed analysis of anal function in patients with SSc, examining those both with and without lower bowel symptoms. The study was approved by the Salford Ethics Committee.

\section{Patients and methods}

\section{PATIENTS}

Only women patients and control subjects were studied. All except two of the 16 patients fulfilled the American Rheumatism Association criteria for SSc. ${ }^{3}$ The two exceptions both suffered from Raynaud's phenomenon and had reduced oesophageal peristalsis, while one of them had sclerodactyly and the other had digital pitting and abnormal nail fold microscopy; clinically, therefore, both were considered to have SSc. Three of the patients had an overlap syndrome, while the other 13 had the limited cutaneous variant of SSc.

Patients were divided into three subgroups on the basis of presence or absence of lower bowel symptoms:

No or minimal lower bowel symptoms-Six patients (median age 44 years, range 27-63; median duration of Raynaud's phenomenon six years, range 3-15).

Constipated -Seven patients (median age 47 years, range 38-68; median duration of Raynaud's phenomenon 20 years, range 5-33). These patients had three or fewer bowel motions per week, with the exception of one patient who passed only small amounts of stool each day with the use of laxatives.

Diarrhoea and faecal incontinence-Three patients (ages 49, 55, and 70 years; duration of Raynaud's phenomenon six, 18, and 50 years). Two of these patients had bacterial overgrowth and were receiving treatment with cyclical antibiotics.

Information regarding upper gastrointestinal symptoms and previous upper gastrointestinal investigations, previous abdominal or anorectal surgery, and drug treatment was documented for each patient.

Results were compared with those obtained in 11 healthy control subjects (median age 53 years, range 29-71).

ANORECTAL MANOMETRY

Anorectal manometry was performed with the patient in the left decubitus position. Pressure data were recorded via an eight lumen, polyvinylchloride, water perfused catheter (Arndorfer Medical Specialities, Greendale, Wisconsin, USA). An Arndorfer low compliance pneumohydraulic infusion pump was used to maintain catheter perfusion at a flow rate of $0.2 \mathrm{ml} \mathrm{H} \mathrm{H}_{2} \mathrm{O} / \mathrm{min}$ per lumen. The perfusion ports were arranged circumferentially at $45^{\circ}$ to each other and positioned $5 \mathrm{~cm}$ from the catheter tip. Each perfusion port was attached to a transducer (Viggo-spectramed, Hadfield, Hertfordshire, UK) and the pressure data processed via a Synectics Polygraf preamplifier attached to an interface card in a PC via an optical cable (Synectics Medical, Enfield, Middlesex, UK). A station pull through was performed in $1 \mathrm{~cm}$ steps, beginning in the rectum and continuing through to the anal verge. This allowed acquisition of circumferential pressure data throughout the 
length of the anal canal. With the perfusion ports positioned in the zone of greatest pressure of the anal canal, the patient was requested to perform three voluntary squeezes to assess external sphincter function. The catheter was then repositioned in the rectum with the perfusion ports at $6 \mathrm{~cm}$ from the anal verge and three rapid pulls at rest and during voluntary squeeze were performed. A constant rate mechanical puller was used to pull the catheter at a rate of $1 \mathrm{~cm} / \mathrm{s}$. Three dimensional pressure profiles, vector volumes, and asymmetry scores were calculated automatically from the defined rapid pull through manometric data.

The manometric parameters measured were: mean resting pressure (MRP) in the zone of greatest pressure; 'maximum voluntary squeeze effort' (MVSE) - the average maximum pressure from the three squeezes; radial asymmetry at rest (Rasym) and during squeeze (Sasym)expressed as coefficients of variation, describing the degree of deviation of the eight individual pressure measurements away from a perfect circle; vector volume at rest (Rvv) and during squeeze (Svv).

The radial asymmetry and vector volume parameters provide detailed analysis of segmental anal canal function. ${ }^{4}$

\section{STATISTICAL ANALYSIS}

Manometric data from control and patient groups were compared by a Rank sum test.

\section{Results}

The table summarises the results. Mean resting pressure, maximal voluntary squeeze effort, and squeeze vector volume were lower in patients with SSc than in healthy controls, and differences were significant in the subgroup with constipation. Squeeze asymmetry was greater in constipated patients with SSc than in control subjects. Among the three patients with diarrhoea and faecal incontinence, mean resting pressure was low in two, and the third had marked radial asymmetry-both resting and squeeze. The figure shows the three dimensional pressure profiles, both resting and squeeze, in one of the healthy control subjects

Anorectal manometry in patients and control subjects

\begin{tabular}{|c|c|c|c|c|}
\hline & $\begin{array}{l}\text { Asymptomatic } \\
S S c \\
(n=6)\end{array}$ & $\begin{array}{l}\text { Constipated } \\
S S c \\
(n=7)\end{array}$ & $\begin{array}{l}\text { Diarrhoeal } \\
\text { incontinent SSc } \\
(n=3)\end{array}$ & $\begin{array}{l}\text { Controls } \\
(n=11)\end{array}$ \\
\hline $\mathrm{MRP}(\mathrm{mm} \mathrm{Hg})$ & $\begin{array}{l}39.8 \\
(18 \cdot 3-76 \cdot 2)\end{array}$ & $\begin{array}{l}42^{\star} \\
(7.5-63.9)\end{array}$ & 31.7 & $\begin{array}{l}65.0 \\
(34 \cdot 3-96 \cdot 4)\end{array}$ \\
\hline MVSE (mm Hg) & $\begin{array}{l}70 \cdot 1 \\
(47 \cdot 5-240 \cdot 2)\end{array}$ & $101 \cdot 7^{\star}$ & $103 \cdot 8$ & 136.5 \\
\hline Rasym (\%) & $\begin{array}{l}7 \cdot 4 \\
(2 \cdot 3-53 \cdot 9)\end{array}$ & $\begin{array}{l}10 \cdot 6 \\
(6.9-39.9)\end{array}$ & $\begin{array}{l}10 \\
(7 \cdot 6-17 \cdot 9)\end{array}$ & $\begin{array}{l}8 \cdot 7 \\
(3 \cdot 8-12 \cdot 1)\end{array}$ \\
\hline Sasym (\%) & $\begin{array}{l}7.9 \\
(2.9-19.4)\end{array}$ & $11 \cdot 8^{\star \star \star \star}$ & 11.4 & $6 \cdot 5$ \\
\hline $\operatorname{Rvv}\left(\mathrm{cm}^{3}\right)$ & 258 & 266 & 80 & $\begin{array}{l}377 \cdot 0 \\
(109-577)\end{array}$ \\
\hline $\operatorname{Svv}\left(\mathrm{cm}^{3}\right)$ & $\begin{array}{l}453 \\
(121-2061)\end{array}$ & $\begin{array}{c}609 \star \star \star \\
(399-716)\end{array}$ & $\begin{array}{l}212 \\
(142-711)\end{array}$ & $\begin{array}{l}885 \cdot 0 \dagger \\
(452-1322)\end{array}$ \\
\hline
\end{tabular}

SSc $=$ Systemic sclerosis; $\mathrm{MRP}=$ mean resting pressure $; \mathrm{MVSE}=$ maximun voluntary squeeze effort; Rasym = radial asymmetry at rest; Sasym = radial asymmetry during squeeze; Rvv = vector effort; Rasym $=$ radial asymmetry at rest; Sasym = ra
volume at rest; $S v v=$ vector volume during squeeze. volume at rest; $\mathrm{Svv}=$ vector volum
tResults from 10 controls only.

${ }_{\star} \mathrm{p}<0.02$ versus controls; ${ }^{\star \star \star} \mathrm{p}=0.003$ versus controls; $\star \star \star \star \mathrm{p}=0.0008$ versus controls. and in one of the patients with diarrhoea and faecal incontinence.

Gastrointestinal dysmotility, gastro-oesophageal reflux, or both, had been demonstrated in nine of the 10 patients with lower bowel symptoms (one patient with normal barium swallow did, however, complain of dysphagia) and in three of the six who were asymptomatic for lower bowel symptoms. Of the three other patients, two had no dysphagia, while the third had dysphagia that had not been investigated.

Among the group asymptomatic for lower bowel symptoms, one had undergone caesarean section, one a laparoscopic sterilisation, and one an appendicectomy. Two of the constipated group had undergone hysterectomy, one an ovarian cystectomy, and one repair of a vaginal prolapse. Two of the patients with diarrhoea and faecal incontinence had undergone hysterectomy, and one an appendicectomy.

Four patients were taking nifedipine: three were asymptomatic and one had diarrhoea and faecal incontinence.

\section{Discussion}

Our results confirm earlier reports of anorectal dysfunction in patients with SSc, and extend these observations by providing a detailed analysis of segmental anal canal function in a cohort of patients with SSc subdivided on the basis of lower bowel symptoms. Statistically significant deficits in segmental anal canal function were found in those patients with SSc who had constipation, who had a longer duration of Raynaud's phenomenon than the asymptomatic group. The trend towards lower pressures, lower vector volumes, and greater asymmetry in patients with SSc who lacked lower bowel symptoms is suggestive of subclinical dysmotility in these patients. Only three patients with diarrhoea and faecal incontinence were studied, so it is difficult to draw conclusions about this subset, especially as two were receiving treatment for bacterial overgrowth. However, as two had low mean resting pressures, and the third had marked asymmetry, it seems likely that this patient group also has significantly impaired anorectal function.

Nifedipine may affect smooth muscle function, and this may have influenced results in the asymptomatic group. However, none of the constipated group was receiving calcium channel blocking drugs.

While our study did not aim to correlate upper and lower gastrointestinal features of SSc, we noted nonetheless that all but one of the patients with lower bowel symptoms had documented upper gastrointestinal dysmotility, as did three of the six asymptomatic for lower bowel problems.

Most of the previous studies examining anal function in SSc have concentrated on patients with lower bowel symptoms. Hamel-Roy et al compared oesophageal and anorectal motility in SSc. ${ }^{5}$ Thirteen of their 15 patients with SSc were constipated, one was faecally incontinent, and one had no lower bowel symptoms. The amplitude of the rectoanal inhibitory reflex 

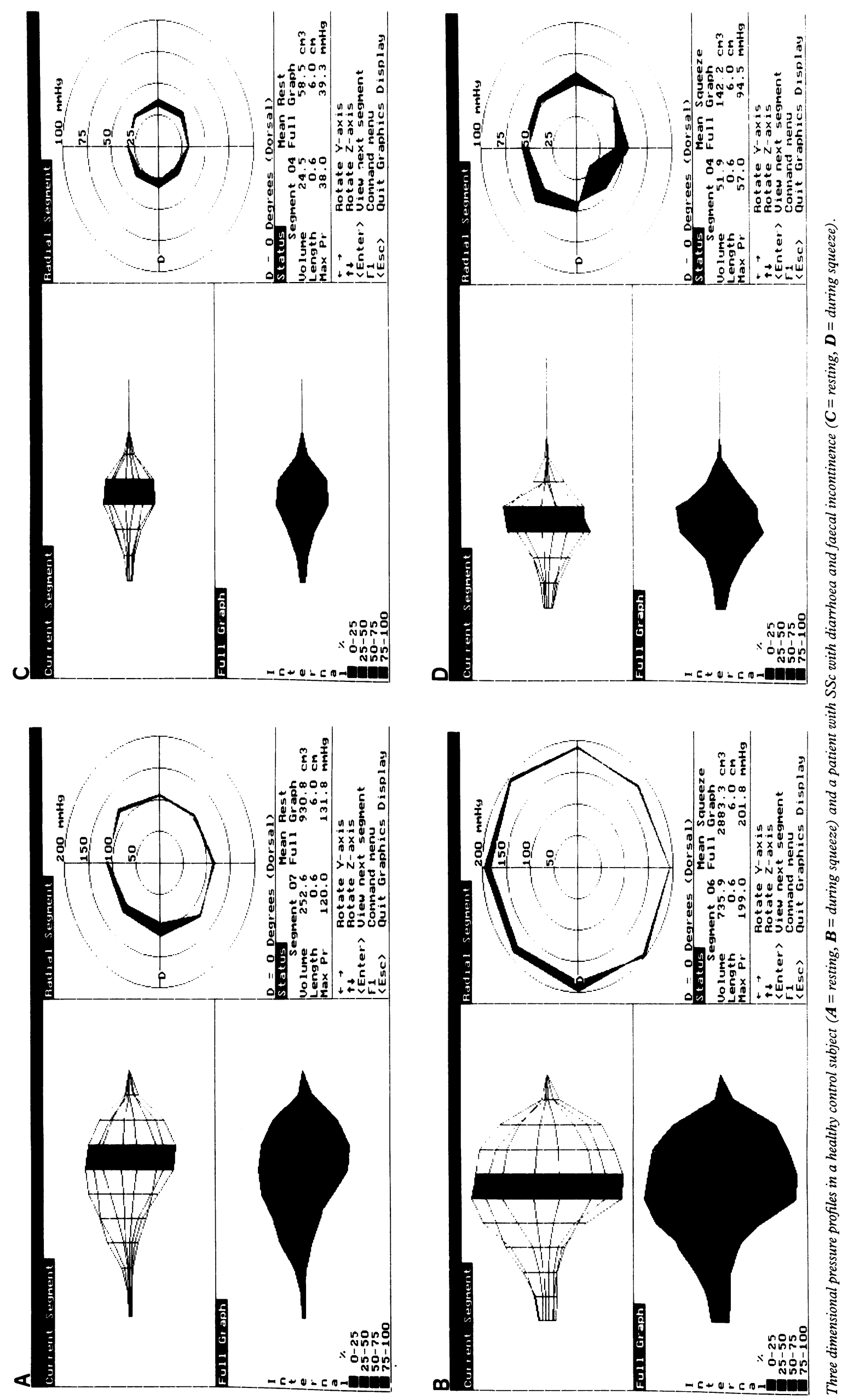
(the reflex relaxation of the internal sphincter induced by rectal distension) was lower in the patients with SSc than in control subjects, and there was a correlation between this and the amplitude of lower oesophageal sphincter relaxation, implying anorectal in addition to oesophageal dysmotility in this group of patients. Patients with SSc also had a reduced resting pressure at the internal anal sphincter compared with controls.

Nine of the 17 patients studied by Chiou et $a l^{6}$ were faecally incontinent, and were therefore clinically disparate from those of the earlier study. Reduction in the maximum basal (resting) pressure in the anal canal was found in patients with SSc compared with control subjects, and 12 patients lacked the rectoanal inhibitory reflex. Six of the eight asymptomatic patients had manometric abnormalities suggesting subclinical dysmotility.

In another study examining mainly patients with faecal incontinence (seven of eight patients had faecal incontinence and four also had rectal prolapse), resting pressures were decreased in all patients, particularly so in those with prolapse. ${ }^{7}$ Squeeze pressures were also less than in controls, and both rectal capacity and rectal compliance were markedly reduced.

Basilico et al studied six patients with SSc who had constipation and eight with normal bowel habit. ${ }^{8}$ Resting pressures in the anal canal were reported to be significantly less than in controls only in the subgroup with constipation, though results suggested that there was a trend towards abnormality in the asymptomatic SSc group.

Finally, a recent report describing two patients with SSc and faecal incontinence suggested selective involvement of the internal sphincter in patients with SSc. ${ }^{9}$ Both patients had a low anal resting pressure and a thin sphincter on ultrasound.

Despite the different methodologies used in the anorectal manometry among these different studies, all reported anorectal dysfunction. Our study is the first to examine segmental anal canal function in patients with SSc and the abnormalities we have described contribute additional information to the understanding of the pathophysiology of anorectal dysfunction in this patient group.

The aetiology of this segmental deficit in anal function in patients with SSc is not known. It has been suggested that neural dysfunction is the initial gastrointestinal lesion in patients with SSc. ${ }^{1}$ Autonomic dysfunction is a recognised feature of $\mathrm{SSc}^{1011}$ and neuropathy of the enteric nervous system has been suggested. ${ }^{12}$ Neuropathy could result either from vascular involvement of the vasa nervorum, in keeping with the suggestion that SSc is primarily a disease of the vasculature, ${ }^{13}$ or from nerve fibre compression as a result of excessive deposition of collagen. ${ }^{14}{ }^{15}$ An initial neuropathy could lead to smooth muscle dysfunction, followed by atrophy, followed finally by muscle fibrosis. ${ }^{1}$ In a postmortem study, muscle atrophy, fibrosis, or both, was found in the large bowel in 21 of 54 patients with SSc, though the anal canal was not specifically reported upon. ${ }^{16}$ From our study it is not possible to determine which of these processes (neuropathy, smooth muscle dysfunction, smooth muscle atrophy, or fibrosis) is responsible for the abnormalities demonstrated. All may contribute, and any of these processes could be envisaged to cause an asymmetric dysfunction.

Does the ability to detect subclinical anorectal dysfunction in patients with SSc have any clinical consequence? While early detection would allow trials of treatment before the stage at which irreversible atrophy and fibrosis have occurred, unfortunately to date there is no satisfactory treatment of SSc. Early detection would therefore simply allow 'symptomatic' treatment at an earlier stage.

Careful assessment of anal canal function may be important in attempts to elucidate further the pathophysiology of the anorectal abnormalities. This is important in a disease for which the pathogenic mechanisms remain poorly understood. Ours was a cross-sectional study, and what would now be of interest would be a larger scale and prospective study, examining anorectal function over time in a cohort of patients with SSc. In patients with subclinical involvement, evaluation could include assessment of anorectal manometry before and after the use of prokinetic agents, as has been performed in the oesophagus ${ }^{17}$ and the colon. ${ }^{18} \mathrm{~A}$ careful prospective study would provide further information on the rate of progression of the anal dysfunction and its correlation with symptoms.

1 Sjogren $\mathrm{R}$ W. Gastrointestinal motility disorders in scleroderma. Arthritis Rheum 1994; 37: 1265-82.

2 Abu-Shakra M, Guillemin F, Lee P. Gastrointestinal manifestations of systemic sclerosis. Semin Arthritis Rheum 1994; 24: 29-39.

3 Subcommittee for Scleroderma Criteria of the American Rheumatism Association Diagnostic and Therapeutic Criteria Subcommittee. Preliminary criteria for the classification of systemic sclerosis (scleroderma). Arthritis Rheum 1980; 23: 581-90

4 Perry R E, Blatchford G J, Christensen M A, Thorson A G Attwood S E A. Manometric diagnosis of anal sphincter injuries. Am f Surg 1990; 159: 112-6.

5 Hamel-Roy J, Devroede G, Arhan P, Tetreault L Duranceau A, Menard H. Comparative esophageal and anorectal motility in scleroderma. Gastroenterology 1985; 88: $1-7$.

6 Chiou A W, Lin J, Wang F. Anorectal abnormalities in progressive systemic sclerosis. Dis Colon Rectum 1989; 32: progressive

7 Leighton J A, Valdovinos M A, Pemberton J H, Rath D M, pamilleri $M$. Anorectal dysfunction and rectal prolapse in progressiv.

8 Basilisco G, Barbera R, Vanoli M, Bianchi P. Anorecta dysfunction and delayed colonic transit in patients with progressive systemic sclerosis. Dig Dis Sci 1993; 38 1525-9.

9 Engel A F, Kamm M A, Talbot I C. Progressive systemic sclerosis of the internal anal sphincter leading to passive faecal incontinence. Gut 1994; 35: 857-9.

10 Sonnex C, Paice E, White A G. Autonomic neuropathy in systemic sclerosis: a case report and evaluation of six patients. Ann Rheum Dis 1986; 45: 957-60.

11 patients. Ann Rheum Dis 1986, 45: $957-60$. Autonomic neuropathy in systemic sclerosis. Ann Rheum Autonomic neuropathy

12 Greydanus M P, Camilleri M. Abnormal postcibal antral and small bowel motility due to neuropathy or myopathy and small bowel motility due to neuropathy or myopathy

13 Campbell P M, LeRoy E C. Pathogenesis of systemic sclerosis: a vascular hypothesis. Semin Arthritis Rheum 1975; 4: 351-68.

14 Di Trapani G, Pocchiari M, Masullo C, Albanese A, Tulli A. Peripheral neuropathy in the course of progressive systemic sclerosis. Ital f Neurol Sci 1982; 4: 341-8.

15 Di Trapani G, Tulli A, La Cara A, Laurienzo P, Mazza S, David P. Peripheral neuropathy in course of progressive systemic sclerosis. Acta Neuropathol 1986; 72: 103-10. 
16 D'Angelo W A, Fries J F, Masi A T, Shulman L E. Pathologic observations in systemic sclerosis (scleroderma). Am $f$ Med 1969; 46: 428-40

17 Cohen S, Fisher R Lipshutz W, Turner R, Myers A Schumacher R. The pathogenesis of esophageal dysfunction in scleroderma and Raynaud's disease. 7 Clin

Invest 1972; 51: 2663-8.
18 Battle W N, Snape W J, Wright S, et al. Abnormal colonic motility in progressive systemic sclerosis. Ann Int Med 1981; 94: 749-52. 